\title{
Internet of Things-Enabled Crop Growth Monitoring System for Smart Agriculture
}

\author{
Hongyu $\mathrm{Hu}$, Department of Computer Information Management, Yongzhou Vocational and Technical College, China \\ Zheng Chen, College of Computer and Information Science, Hunan Institute of Technology, China \\ Peng Wen Wu, College of Computer and Information Science, Hunan Institute of Technology, China
}

\begin{abstract}
In order to solve the problems of high cost and difficult management of traditional agricultural planting, internet of things (IoT) technology was applied to realize real-time detection and intelligent management of crop growth and remote control of equipment, and change the traditional agricultural planting mode. The research results show that in MyEclipse development environment, using B/S (Browser/Server) architecture, Java and JavaScript language to design, Tomcat built server to publish information and complete the function of data storage and query, users can access the monitoring center in the local area network (LAN). When the detected data exceed the set threshold range, the control instructions issued by the monitoring center are transmitted to the main control chip through ethernet, and then the switching operation of the relay is controlled. The real-time monitoring of crop growth environment can be realized.
\end{abstract}

\section{KEYWORDS}

Crop Growth Monitoring, Internet of Things, Smart Agriculture, Wireless Sensor Network

\section{INTRODUCTION}

Agricultural production in China is small in scale and the cultivated land is highly dispersed (Yang, Liu, Long, \& Qiao, 2015). For a long time, it relies on backward production tools for simple reproduction. Agricultural production is the core pillar industry of the country, which is not only related to people's food and clothing, but also has a very important impact on the international status of the country (Wilkinson \& Rocha, 2009). For China, there is a problem of large population and small cultivated land. How to improve the utilization rate and output of unit cultivated land is a key problem facing China's agriculture (Zheng \& Yingbanditpong, 2019). At the same time, with the rapid growth of China's economy, a series of problems have gradually emerged, such as resource shortage, soil degradation, environmental degradation etc. For China's agriculture, in the production process of recent decades, the efficiency and management level of agricultural production has not been improved because of backward production technology, lagging infrastructure, and low scientific and technological content for a long time. Agricultural Internet of Things (IoT) is to use various sensors, RFID, visual terminals and other sensing equipment to widely collect the information in the fields of any medium, provided the author of the original work and original publication source are properly credited. 
farmland planting, gardening, livestock breeding, aquaculture, product logistics, etc.; establish data transmission methods, make full use of wireless sensor network, mobile communication network and Internet and other modern means to achieve reliable transmission of agricultural information (Ray, 2017). Finally, the agricultural information will be integrated and processed, and the agricultural automatic production, optimized control, intelligent logistics, and e-commerce will be realized through the terminal, so as to improve the intensive feature, high-yield, and safety of agricultural production (M. Chen, Mao, \& Liu, 2014).

Figure 1, presents the basic architecture of Wireless Sensor Networks (WSNs) and Internet of Things (IoT) based smart agriculture system. The deployed sensors continuously monitors weather conditions, crop and soil conditions and transfers the sensed parameter to the cloud platform through gateway. The cloud system stores the data in database and user can access data from anywhere at any time for its analysis in real time (Amit Sharma, Singh, Sharma, \& Kumar, 2019).

With the rapid development of China's agricultural science and technology, IoT emerges as the times require in the development of "informatization" in the new era, which is an important part of information technology. As the name suggests, IoT is to use the network to connect sensors and controllers, form the connection between things and the network, and realize intelligent management and remote control (Subashini, Das, Heble, Raj, \& Karthik, 2018). The first generation of agricultural IoT technology relies on large-scale basic agricultural equipment. The switch of this equipment mainly relies on manual operation to realize the power supply and power-off of the motor (Awan, Ahmed, \& Hashim, 2019). With the continuous development of technology, the second generation of agricultural IoT has added corresponding sensor equipment and control terminal, which can view the corresponding sensor value and realize the control of agricultural equipment. However,

Figure 1. General architecture of smart agriculture based on WSNs and loT

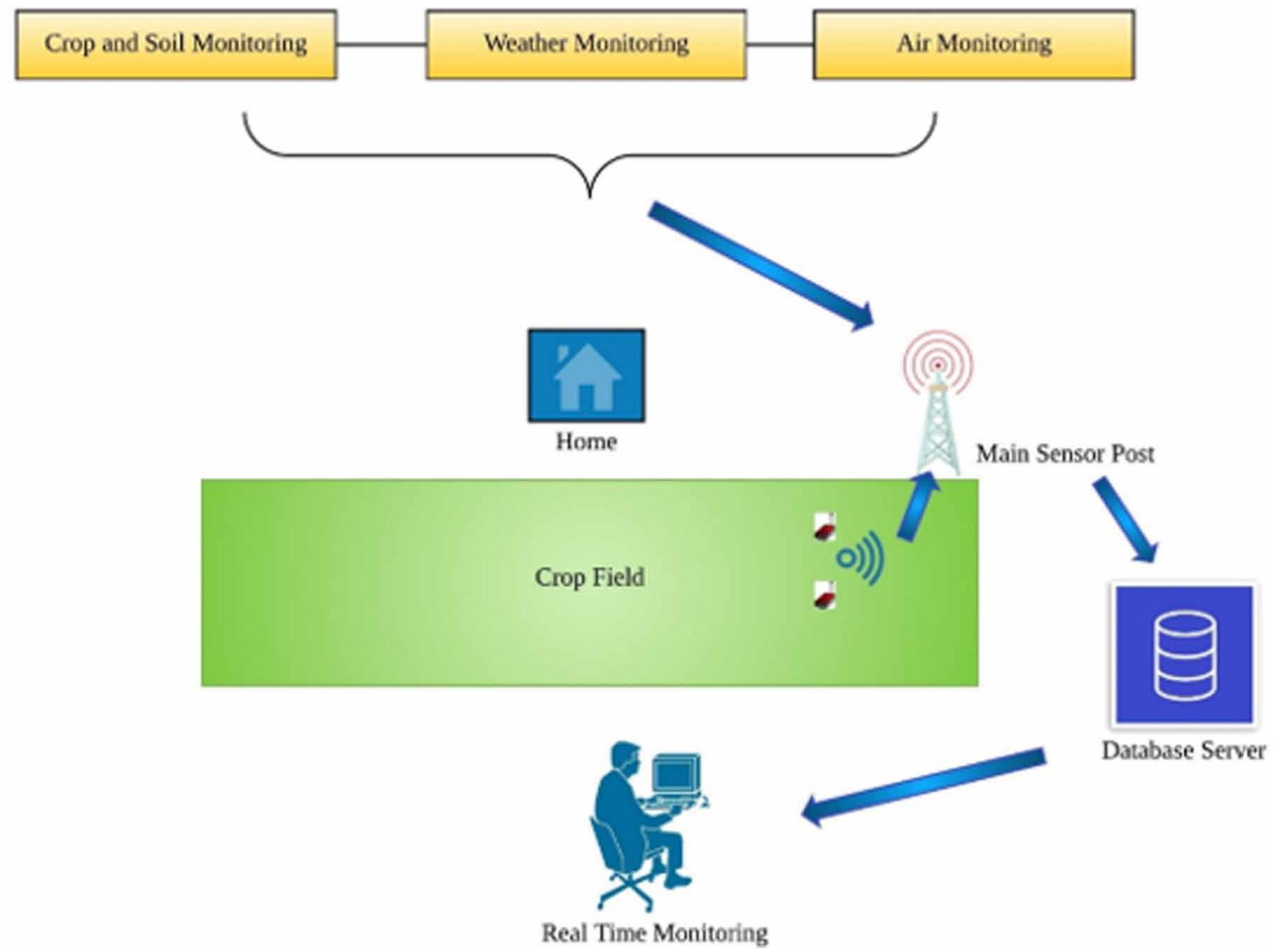


the disadvantage of the whole system is that the data is transmitted by wire. To realize the remote transmission, it is more difficult, the line is long and easy to consume resources, and it is very easy to fail (Kumar, Sharma, Kumar, \& Sharma, 2020).

The IoT technology is applied into agricultural production and scientific research, which can change the mode of agricultural production and management level, accelerate the development of agricultural modernization in China, and achieve smart agriculture. IoT technology can be widely used in many agricultural fields, including remote monitoring and fine management of crop production process, monitoring of agricultural aquaculture, traceability of agricultural products safety, etc. It can be seen that intelligent agriculture based on IoT is a change of modern information technology in the field of agriculture. Under the influence of information technology such as IoT, traditional agriculture will have great changes in a short period of time. The application of IoT technology in agriculture can help farmers professionally manage and plant scientifically, improve the comprehensive benefits of products, and realize the standardization, digitalization, and networking of agricultural production (Ashutosh Sharma \& Kumar, 2016).

The rest of the paper has been organised in the following sections: Second section constitutes the literature review which is useful for understanding of concept of IoT toward agriculture. Section three consists of system model design which includes analysis of requirements of components. Section 4 consists of software analysis used for the agriculture application. Section 5 includes the results and discussion. The last section constitutes conclusion of the work.

\subsection{Motivation and Contribution}

Recently, agriculture domain is mostly explored based on WSNs application in order to improve traditional farming methods. The introduction of MEMS technology enables the use of small and low cost effective sensors. These small size node along with the advantages of self-organization, scalable network enables WSNs a potential technology for achieving agricultural automation. There are certain limitation of WSNs such as limited battery, small memory and limited computation and these limitations brings many challenges in designing application for smart agriculture using WSNs. The solution of farming based on WSNs needs to be of low cost such that the application can be affordable by users. The increasing population also give rise to the demand of food grains in China. It is noticed from the recent report that the growth of population is more comparatively with the growth of food production. Also, China is a country that exports its most of its food grains and therefore researchers investing on using advanced technologies for increasing the production. In this article we have studied various WSNs technologies and their potential for the development of smart agriculture application. This article mainly focuses on decreasing the water consumption along with increase in productivity for small farms.

\section{LITERATURE REVIEW}

In research field, there exists lot of work that focused on application of WSNs and IoT technology in agriculture field. Some of the system uses WSNs tools for collection of ground and environmental parameters and IoT platform for the interconnection and to generate various projects. In our study we are focused on the articles that are related to this subject of smart agriculture utilizing sensor networks and internet of things (IoT). The articles have been studied either to get knowledge about their basic purpose and its architecture or the basis of hardware used.

(Khan \& Ismail, 2018) research showed that IoT had changed the field of agriculture, enabling farmers to deal with a large number of problems they face. In order to support IoT to improve the cultivation, yield, and quality of crops, it is necessary to enhance security through appropriate access control, data confidentiality, and user authentication (Khan \& Ismail, 2018). Agriculture needs technical skills to enhance organizational capacity, perform agricultural functions, and solve problems. The right support infrastructure can be developed with the right Internet availability and 
connectivity, and review IoT applications, including low-power wireless sensors, better connectivity, operational efficiency, and remote management. (Anandkumar, Kalaiarasan, \& Balakrishnan, 2018) proposed a "soil analysis and irrigation system based on IoT" to help farmers reduce labor and increase production and use IoT to try to automate irrigation, and farmers can even control the power of the motor remotely (Anandkumar et al., 2018).

(Y. Chen, Zhao, \& Zhou, 2018) studied and analyzed the key technologies of IoT in intelligent agriculture, and introduced the application of IoT technology in agricultural planting system. They also pointed out that, in the process of transformation to modern agriculture, China's traditional agriculture was facing the problems of ensuring the quality of agricultural production, adjusting the structure of agricultural industry, improving the production efficiency, and solving the problems of low resource utilization and environmental pollution, so as to meet the sustainable needs for agricultural development (Y. Chen et al., 2018). Therefore, the research on intelligent agricultural technology is imperative. (Lin, Li, Feng, \& Zou, 2018) proposed a new intelligent greenhouse cultivation system, which could not only liberate the labour force of farmers, but also break the restrictions of disastrous weather impact, pest outbreak and other adverse environment (Lin et al., 2018). The system has been put into use in Southeast China, including four functional parts: environmental parameter collection, data transmission, information processing, data adjustment, and feedback control. These useful data from IoT sensors can be used to evaluate plant growth and predict the amount of liquid fertilizer to minimize resource waste (Rashid \& Bapujee, 2019).

(Riquelme et al., 2009) present a system which states that it is not easy for small scale farmers to accomplish the policies of green house because of their cultural and economic conditions. Therefore, considering this problem they have designed a system for horticulture production that consists of sensor nodes and modules for substantially maintaining the greenhouse. The information which is required for the operation of their system are the plant and environmental parameters (Riquelme et al., 2009). There exists more studies based on similar hardware components presented by (López et al., 2011), (Bontsema, Van Henten, Gieling, \& Swinkels, 2011), (Parameswaran, Zhou, \& Zhang, 2013). Their systems mainly focused on monitoring pepper crop field, rose and palm oil plantation.

(Chappell, Dove, van Iersel, Thomas, \& Ruter, 2013) proposed a WSNs based platform for the irrigation control implemented to the greenhouse (Chappell et al., 2013). Their study presents the evaluation of soil and environmental parameters which are visible form a web based application and the generated information can be shared in the form of CSV files. (Tanumihardja \& Gunawan, 2015) presented a system based on WSNs with the objective of correct administration of water levels (Tanumihardja \& Gunawan, 2015). Another system based on WSN and IoT which is programmed for analysing the soil moisture in caladium greenhouse. They have utilized the hardware and software of libelium and the collected information is analysed through web application. One of the systems is utilizing WSNs and IoT for a programming irrigation in coffee crop during dry season (Myint, Gopal, \& Aung, 2017). (Pang, Chen, \& Zheng, 2011) presented an article which emphasizes food safety, more importantly the quality of agriculture products (Pang et al., 2011). One more study present the importance of agriculture products for quality ford (Biccario, Annese, Cipriani, \& De Venuto, 2014). Their objectives of the project are to present the food of good quality. Their system based on WSNs for the measurement of environmental and soil parameters by utilizing Arduino devices and ZigBee modules for communication. Guillermo et al. proposed a protocol based on long range wide area network (LoRaWAN) and aerial image processing (Guillermo, García-Cedeño, Rivas-Lalaleo, Huerta, \& Clotet, 2018). Their system consists of WSNs which are connected to the irrigation controller in combination with image processing. The system is capable of providing ground data, weather conditions, flow of water and its pressure. The sensed data of their system is available for display through mobile application. There are many studies documented for the agricultural technologies, among which mostly depends on spectrometry principle, image based analysis and many other related studies. (Cambra, Sendra, Lloret, \& Garcia, 2017) developed an application based on WSNs and IoT for the precision farming (Cambra et al., 2017). Their study mainly focused on the management of 
collected information with cloud computing. The management of information through cloud is done in such a way that it can be applied for cacao crop for irrigation control. The drawback of their study is that it does not consist of any experimental analysis. (Guillermo et al., 2018) presents another system where IoT is utilized for the production of Cacao (Guillermo et al., 2018). Their system through deployed sensors collects information and monitors temperature, humidity, oxygen, moisture and carbon dioxide. The collected variables are then analysed for the fermentation practice of cacao beans.

( $\mathrm{Rad}$ et al., 2015) has proposed a WSNs based architecture for the collection and analysis of agricultural environment for potato farming. Their design help farmers to identify irrigation and fertilizer requirements. The studies related to the use of WSNs, users can monitor the soil related parameters. The sensor nodes were deployed under soil for measuring quantities and communicating measured information using relay nodes using effective routing protocols which provides very low duty cycle, hence increasing the life span of monitoring system. The advancement in the technology of WSNs and IoT has gained attention of various researchers across the world. The extensive contributions can be found from researchers in the field of agriculture through WSNs and IoT, but there found very little evidence can be found on real time monitoring of crop through video surveillance. This article is an attempt for expanding the research by proposing a real time agriculture monitoring system through WSN, video surveillance and IoT. The system is designed by considering small and large scale crop producers with the objective of presenting a user friendly interface (Ashutosh Sharma \& Kumar, 2017).

\section{SYSTEM SCHEME DESIGN}

In terms of agricultural planting, China relies on traditional agriculture for a long time to produce. The long-term traditional agricultural production mode makes agricultural production inefficient and management backward. In order to solve this problem, an agricultural monitoring system (SASIOT) project based on IoT is proposed and implemented, which has the advantages of real-time monitoring and intelligent management. For intelligent agriculture, the main monitoring parameters of farmland environment are soil temperature, soil humidity, environmental temperature, environmental humidity, rainfall, wind speed, $\mathrm{CO}_{2}$ concentration, and water environmental $\mathrm{pH}$ value and so on. For the collection of farmland environmental parameters, due to the complexity of farmland geographical conditions and the diversity of collection parameters, many sensors need to be deployed in the field, and these sensors usually need to be moved in the field, and they cannot work normally in the face of energy exhaustion and other faults. In the past, the design of monitoring system was mainly based on wired mode. For large areas of farmland, its application had limitations and could not meet the dynamic requirements of the network. Therefore, the research and design of an agricultural monitoring system with real-time monitoring and intelligent management will be of great significance for promoting the upgrading and reforming of traditional agriculture in China, improving the quality of agricultural products, and improving the efficiency and management level of agricultural production. After putting the system into practice, it can greatly improve the quality of agricultural products, improve the efficiency of agricultural production and management level, and play an important role in the development of agricultural economy in this area.

Figure 2 presents the intelligent agriculture for real time monitoring of agricultural field where deployed sensor nodes collects the field parameters and transmits the sensed information to the user through gateway. The proposed system also integrates cameras for providing high definition video for real time monitoring of the field. This information can be processed by the end user in real time through gateway node. Based on the analysis of the actual situation of intelligent agriculture, the following requirements are obtained:

1. The data acquisition module. It is mainly for the collection of agricultural environmental parameters, including environmental temperature, environmental humidity, light intensity and other parameters. The collected data is integrated and processed by STM32. 


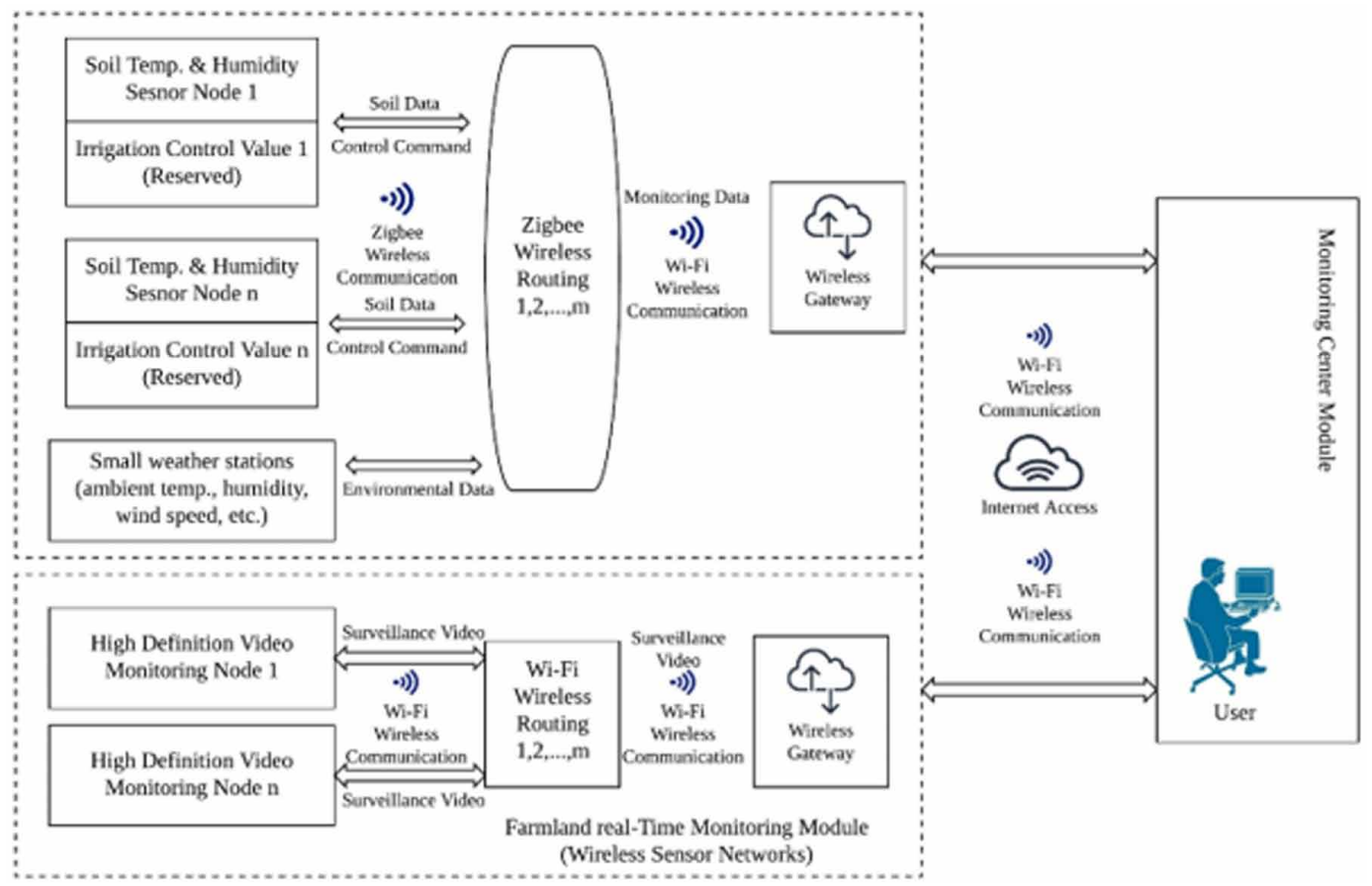

2. The software design of the control module. It is mainly that the MCU receives the signal from the monitoring center and controls the equipment, including the control of spray and drip irrigation equipment.

3. The software design of the serial port module. STM32 transmits the collected data to the $3 \mathrm{G}$ module through the serial port.

4. Data transmission module. 3G module is used to transmit the data collected by the sensor to the server, and the data is transmitted through wireless equipment.

5. Ethernet module. Use DM9000 network card chip to transmit the control signal sent from the monitoring center to the single chip microcomputer control system.

6. The software design of the monitoring center. It mainly uses the server built by Tomcat to release information, including the database design and the interface design of the monitoring center. The design of database includes data storage, equipment management, and user management; the interface of monitoring center is mainly the medium of information interaction between user and system, which is more convenient for user management.

\subsection{Adopted Methodology}

The problem of monitoring small and large scale fields is identified and such that the process is initiated with for gathering the information at ground level. The area around 2 hectare is analysed with the purpose of determining physical, chemical and environmental parameters. The development methodology of our system for monitoring the agriculture fields consists of sensor deployment and IoT platform. The architecture represents integration of WSNs and IoT and the overall processing is carried out in three layers; deployment layer, service layer and application layer as presented in Figure 3.

The proposed architecture consists of three operational layers and is efficient in monitoring small and large scale fields. The system is capable of collecting information of soil and climate variables, storing and processing it using some data mining techniques for its analysis and visualization through 


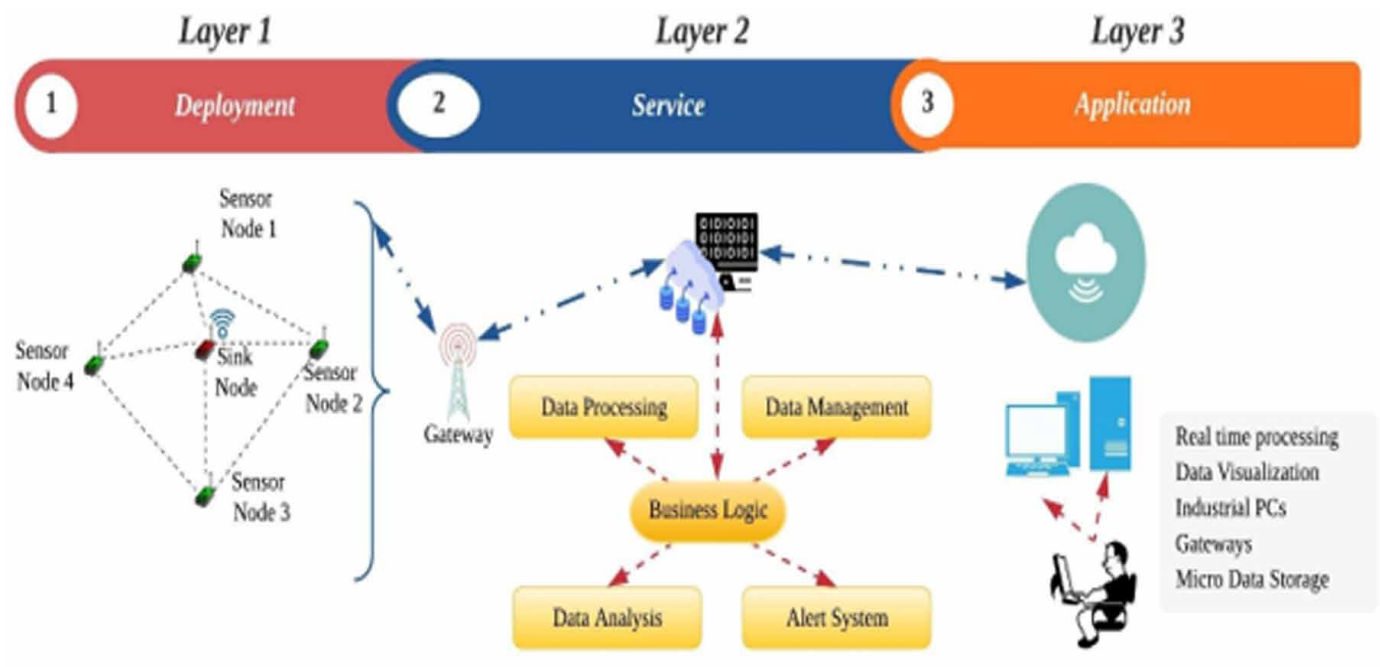

IoT based application. The proposed system allows farmer to visualize the collected information of the field and weather in real time. In case of any event the system will generate an alert message and forward alert instantly through emails or text notification. The purpose of opting WSNs and IoT technology is to monitor the crop in real time for presenting optimal and timely solution of agriculture issues. The operation layers with working modules are described below.

\subsection{Deployment Layer}

This layer is responsible for collecting the soil and environmental parameters and transferring them through gateway or sink note to cloud platform (Anitha \& Acharjya, 2018). The nodes mentioned below are utilized for monitoring soil and environmental parameters and they are categorized with their operating range presented in Table 1.

Each sensor collects and transmits the information to coordinator node, which further transmits the sensed data to the cloud platform through GSM module. The hardware components of the coordinator node are described in Table 2.

The WiFi networking utilizes low range low power consumption wide area network and incorporates as the physical layer. The gateway node through WiFi networking module receives all the sensed information and transmits to the database server for its analysis and storage.

Table 1. Sensor nodes hardware components

\begin{tabular}{|c|c|c|c|}
\hline & \multicolumn{3}{|c|}{ Processing Data } \\
\hline Sensor Model & Measuring Variable & Range & Resolution \\
\hline DHT 22 & Temp. and Humidity & -40 to $80^{\circ} \mathrm{c}$ and $0-100 \%$ & $\begin{array}{c}0.1 \% \text { RH } \\
0.1^{\circ} \mathrm{c}\end{array}$ \\
\hline pH meter & $\mathrm{pH}$ & $1-10$ & Not Specified $^{\text {RH }}$ \\
\hline TSL 2561 & Luminosity & $0-40,000$ lux & 0.1 lux \\
\hline Hydrometer & Soil moisture & $0-100 \%$ & Not Specified \\
\hline
\end{tabular}


Table 2. Coordinator node hardware components

\begin{tabular}{|c|c|c|c|}
\hline \multicolumn{4}{|c|}{ Coordinator Node } \\
\hline Hardware & Model & Specification & Transmit and Receive Power \\
\hline $\begin{array}{c}\text { Microcontroller (Single } \\
\text { board) }\end{array}$ & Sensenut & 32-bit RISC microcontroller & $\begin{array}{c}\text { Rx-17mA } \\
\text { Tx-15mA }\end{array}$ \\
\hline WiFi network Module & SenseNut & $\begin{array}{c}\text { Low power WiFi networking } \\
\text { module }\end{array}$ & $\begin{array}{c}\text { Rx-6.9mA } \\
\text { Tx-12.5mA }\end{array}$ \\
\hline
\end{tabular}

\subsection{Service Layer}

It is responsible for providing the required essentials to user for managing, visualizing and processing the data and sends an alert message in case of any adversary. The interaction among deployment and application layer are carried out in three steps; information management, processing data for analysis and alert generation. These three services are accessed in cloud platform and can be accessed from any location. The working of each module is presented below.

\subsubsection{Information Management}

The sensor collects data of surrounding continuously and it is important to process the generated data in real time. Therefore information management becomes essential as the generated data of large volume needs to be managed for processing. The administrator can manage the network, modify it by adding or deleting some nodes, visualize the collected data, analyse the data in real time and present the data in the form of statistical graphs and maps. Additionally, the previous records are also available that can be stored back for analysis and comparison of present situation. This interaction between application and deployment layer is achieved by implementing an intermediate layer which is developed using business logic in PHP and JavaScript This module provides the necessary information to the users at right time for decision making.

\subsubsection{Data Processing and Analysis}

This module is responsible for pre-processing of raw data stored as the CSV format for the replication of data, error correction and elimination of duplicate data. The raw data is pre-processed for its transformation using pattern recognition method for its evaluation and interpretation. In this project we have utilized Mlib framework as it provides the benefits of processing much complex data. After the processing of data, system runs distributed computing in order to obtain relevant information and reports. The evaluation of data is carried out using Apache Spark framework as it is the most powerful and efficient tool for big data analytics. The module of analysing and processing information is implemented for improving the data mining process, for obtaining the useful information and to reduce the original size of data.

\subsubsection{Alert Generation Module}

This module works with the highest and lowest value of soil and temperature variables for the monitoring of agriculture. The threshold value for the highest and lowest is fixed and whenever any event causes the threshold to exceed, system generates an alert and transmits to the users through emails or text. The highest and lowest value of each sensor node is specified and pre-defined in system and provides the flexibility of changing them as per the different application requirements.

\subsection{Application Layer}

This layer is responsible for maintaining the interconnection between the user and the IoT devices. Thus, the monitoring operation can be performed through remote locations. The application interface 
offers the real time monitoring of environment, visualization of sensor data, working of each node and their locations and data queries.

The agricultural monitoring system based on IoT integrates the wireless sensor network (WSN) and the wireless video monitoring network with the Internet. The system can be divided into 3 parts according to the three-layer architecture of IoT: the perception layer, the transmission layer, and the application layer. The perception layer is the lowest level of the system, which aims at obtaining agricultural environmental information through a variety of sensor arrays, accomplished by WSNs and wireless video monitoring networks deployed in the fields.

The transmission layer is the middle layer of the system, and the main equipment is the wireless gateway (coordinator gateway). Its main function is to transfer the agricultural environment information acquired by the perception layer to the application layer, which plays a bridge role. The application layer is the highest level of the system, that is, the monitoring center module, which is mainly composed of Web server and database, used for data storage and information distribution (Zhang \& Wang, 2019). The monitoring center is designed with B/S architecture. For agricultural managers and ordinary users, it can not only check agricultural environment information through the monitoring center, including real-time data and historical data of agricultural environment, but also control, manage, and send relevant equipment of the system.

The whole working process of the system is as follows: collecting farmland environmental parameters and real-time monitoring crop growth status by deploying sensor nodes, small weather stations, and high-definition video monitoring nodes in farmland, gathering collected data or field images into wireless gateway (coordinator gateway) and wireless gateway through WSN and wireless video monitoring network (Ruan et al., 2019). The wireless gateway (coordinator gateway) forwards data to the monitoring center for storage. Agricultural managers or ordinary users can log on to the Web server after system registration to get real-time knowledge of farmland environmental parameters and crop growth status. In the front-end interface of the monitoring center, the environmental evolution process can be displayed in the form of histogram, dial and curve. At the same time, the sensing nodes and wireless gateway (coordinator gateway) can be controlled.

\section{DESIGN AND IMPLEMENTATION OF SYSTEM SOFTWARE}

\subsection{Design of System Software Architecture}

Keil MDK is a kind of embedded software, which has powerful C compiler, rich library management, simulation and debugging tools, and a better human-computer interaction environment. KeilMDK contains excellent $u$ Vision 5 integrated development environment and powerful Real View compiler, which provides high-quality development platform for Cortex-M, ARM7, ARM9 and other microprocessors, especially the best development platform for Cortex-M core processor. It supports TCP / IP communication protocol and various applications, including USB device and USB host stack. The execution analysis tool and performance analyzer optimize the program and make the application scope of the software wider.

The system sends the relevant agricultural environment parameters collected by the sensor to the upper computer through $3 \mathrm{G}$ network and stores them. The software design of the monitoring center is mainly to release the information, send the data such as agricultural environment parameters to the database for storage through relevant programs, and the database will monitor the relevant operation of the web server, trigger the corresponding instructions through the receiving and sending processing programs, and control the corresponding equipment.

In terms of system software architecture design, this paper designs according to four information flow directions of data acquisition, transmission, storage and release. The software system mainly develops and designs related programs from three aspects, including the sensing terminal program of the sensing node, the gateway application program of the coordinator 
gateway, and the related program of the monitoring center (Jinbo, Yu, \& Lam, 2018). The system sends the data of agricultural environment parameters collected by the sensing node to the database through related programs for storage. At the same time, the database monitors the relevant operation of the Web server, and triggers the corresponding instructions and control of the sensing node through the receiving and sending processing program. The system software architecture is shown in Figure 4.

\subsection{Sensing Node}

The program design of WSN node mainly includes two main parts, the sensor node program and the gateway node program, which are mainly responsible for the acquisition and transmission of data in the sensing area of farmland. Each node has a unique node address for recording the relevant information of the node. For sensor nodes, after power-on and initialization of the system are completed, each node will collect and send data according to the default configuration. If the node receives the relevant configuration command from the monitoring center, it will be parsed and processed. At the same time, the configuration information will be modified according to the relevant instructions, and work according to the modified configuration information. For example, the sensor node will send the data acquired to the gateway node according to a certain period (Vincent et al., 2019). For gateway node, it is mainly responsible for data transmission between sensor node and monitoring center. After power-on and initialization of the system, both ZigBee module and serial module are monitored in real time. If the data is monitored, data will be parsed. After parsing, data will be written into serial port on the one hand, and data will be sent through zigBee module on the other hand. The flow charts of sensor node program and gateway node program are shown in Figures 5 and 6, respectively.

The TDR-3 is used to design the soil moisture sensor. The conversion of the output voltage to the actual soil moisture content is shown in equation (1):

$$
\theta_{v}=0.0337 \times \text { Vout }^{3}-0.0426 \times \text { Vout }^{2}+0.2008 \times V_{\text {OUT }}-0.0041
$$

$\theta_{v}$ indicates the soil moisture content, and $V_{\text {OUT }}$ refers to the sensor output voltage value.

Figure 4. The system software architecture of agricultural monitoring system

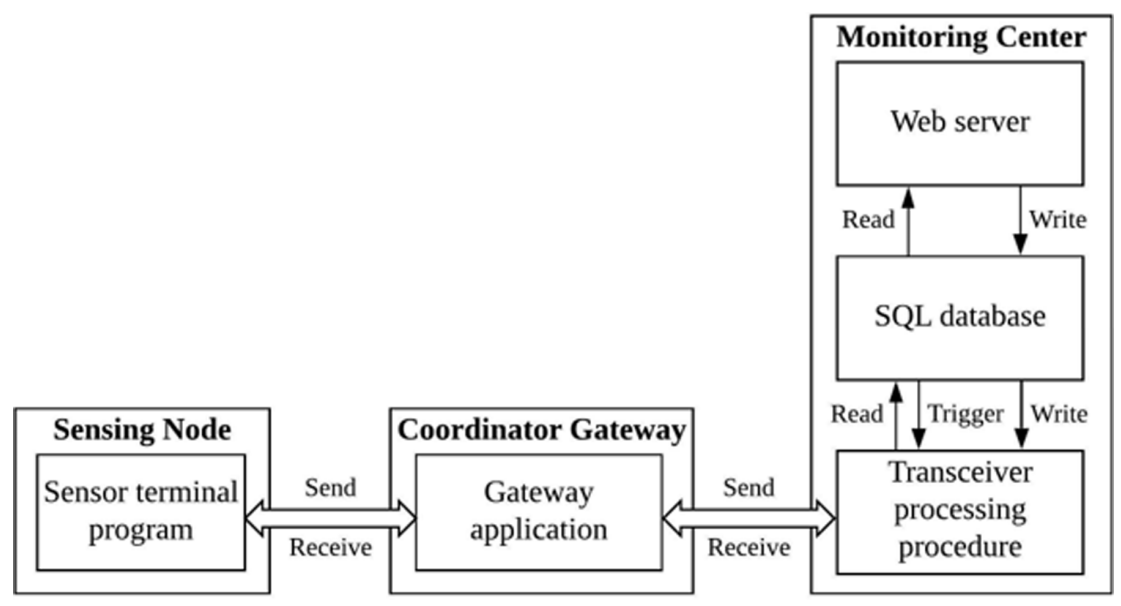


International Journal of Agricultural and Environmental Information Systems Volume 12 •Issue 2 • April-June 2021

Figure 5. Flow chart of sensor node program

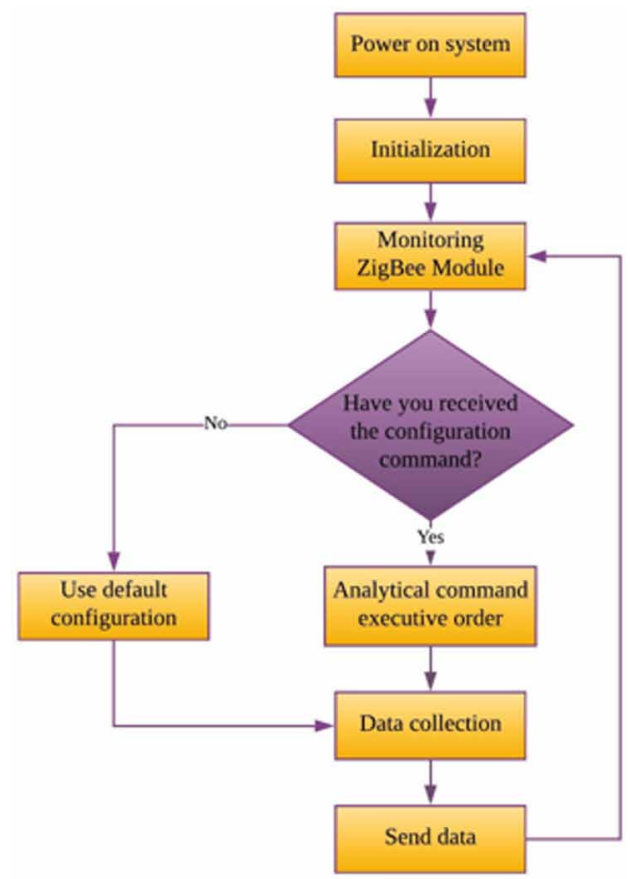

Figure 6. Flow chart of gateway node program

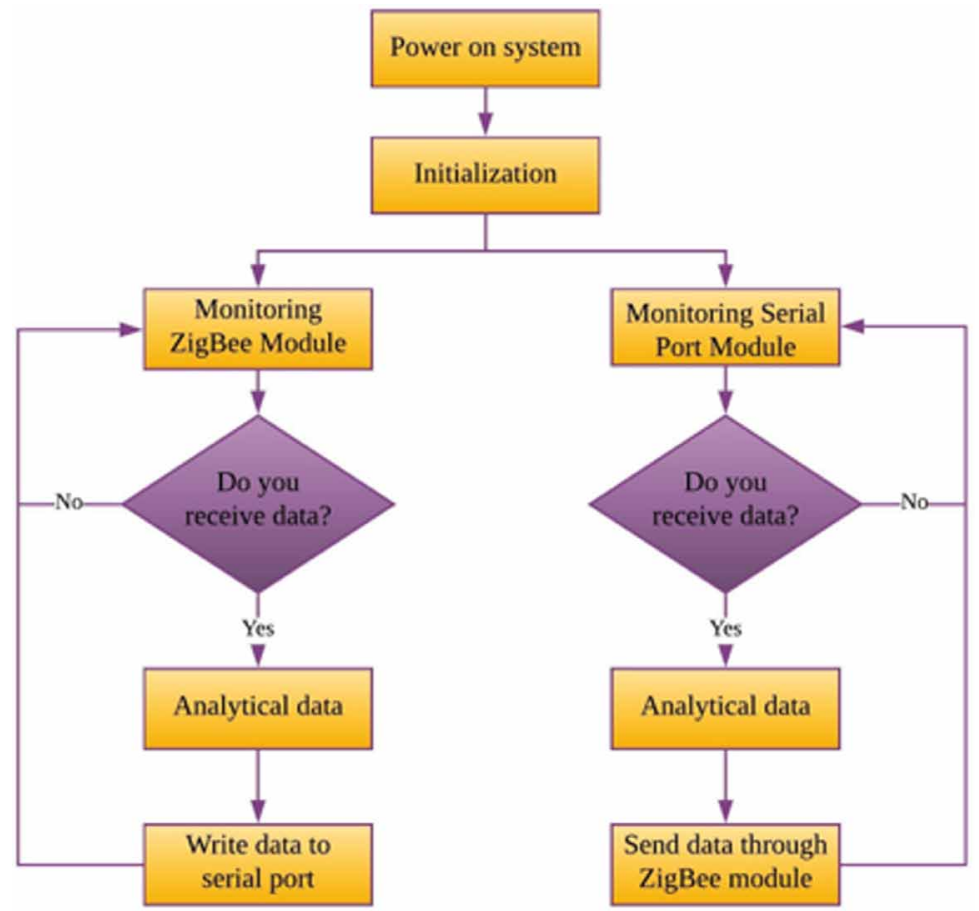


The $\mathrm{CO}_{2}$ concentration sensor is designed with SH-300-ND. The output voltage of the sensor is linearly related to the actual value of $\mathrm{CO}_{2}$ concentration:

$$
Y=\frac{5000}{3} \times X
$$

$\mathrm{Y}$ is the concentration of $\mathrm{CO}_{2}$ and $\mathrm{X}$ is the output voltage of the sensor.

E-201-C-9 type $\mathrm{pH}$ electrode is used to design the $\mathrm{pH}$ sensor for water environment. The output voltage of the sensor is linearly related to the actual value of $\mathrm{pH}$ :

$Y_{1}=\frac{1000}{181} \times X_{1}$

$Y_{1}$ denotes the water environmental $\mathrm{pH}$ value, and $X_{1}$ is the sensor output voltage value of the water environmental $\mathrm{pH}$.

Select the DS-2CD5A24FWD-IZ (H) (S) model high-definition camera as the agricultural environment monitoring node, which can collect real-time video data for the monitored area, and compress the collected video data, while supporting the RS232 interface. The basic format of the downlink message is shown in Table 3.

The header is in a fixed format and has two-byte lengths, namely 0X90EB. Cameras can set their own addresses, ranging from 0 to 255 . For the types of messages, they can be divided into many types, including setting image parameters, responding, taking commands, testing instructions, image data transmission and so on.

\section{SIMULATION RESULTS AND DISCUSSION}

In this section we will discuss monitoring central system for agriculture application. Also, we will discuss the platform used for the implementation of IoT in agriculture.

\subsection{Analysis of Monitoring Center System}

According to the actual needs, MyEclipse is chosen as the development platform of the monitoring center. The platform has abundant functions and can realize all the requirements in the process of program development. MyEclipse development platform, as Elipse plug-in, supports JSP, Javascript, SQL and other related JAVA core development, so as to achieve the purpose of efficient and rapid development of monitoring center. The monitoring center mainly uses JAVA, JSP and HTML to develop and design. In the process of development and design, embedded code is used to complete the relevant operation of the monitoring center Web page. Through HTML (Hypertext Markup Language) based on $\mathrm{B} / \mathrm{S}$ architecture, it has strong interoperability. JAVA is not only a programming language, but also an application development platform (Kiani \& Seyyedabbasi, 2018). It is mainly developed by SUN Company and has three kinds of development platforms. JavaSE is the Standard Version development platform, mainly used to develop desktop and server application software. JavaEE is

Table 3. Downlink message format

\begin{tabular}{|c|c|c|c|c|c|}
\hline Message header & Camera address & Message type & Message length & $\begin{array}{c}\text { Command } \\
\text { parameter }\end{array}$ & CRC check code \\
\hline 2byte & 1 byte & 1 byte & 2 byte & $<(65536-9)$ & 2 byte \\
\hline
\end{tabular}


the enterprise version development platform, mainly used to develop enterprise systems, such as enterprise portal website. As a mobile version development platform, JavaME is mainly used for the development of embedded products and mobile terminals. As a dynamic web page technology, JSP mainly implements related functions through JAVA code and HTML code (Marques \& Pitarma, 2019). It can interact with database through JAVA code and has obvious advantages for the development of dynamic web site. The data statistics and analysis interface can query and analyze agricultural environmental information, including soil temperature, soil humidity, environmental temperature, environmental humidity, rainfall, wind speed, $\mathrm{CO}_{2}$ concentration, water environment $\mathrm{pH}$ value, and other important agricultural environmental information affecting crop growth.

Through the analysis of the data stored in the database, it can directly reflect the changing law of the environmental parameters of the farmland that affect the growth of crops. Through the comparative analysis of these data, the best environmental parameters of the growth of crops can be obtained, so as to facilitate the agricultural managers to know the growth of crops and realize scientific planting and management.

In order to query the historical data in the database, it is necessary to connect the database. Only when the database connection is completed can the database be accessed. For query results, it is supposed to use xx.nextO to make circular judgment. If there is data, output the query results through getString $\mathrm{XxxO}$ statement. The purpose of rs.nextO in the first query is to find the corresponding node address as the condition of the second query. The second query of rs2.nextO is mainly based on the corresponding node address as the query condition, to realize the query of the corresponding environmental parameters of the node address. When the user submits the query command, the system will query the database through the above program and return the query results to the client (Köksal \& Tekinerdogan, 2019). The historical information for the node address of 080F0205 is shown in Table 4.

The display after data query is completed by JFreeChart, which is an open-source Java chart drawing tool. It can generate many kinds of charts, including curve, dial, and histogram, which can be used in different architectures of C/S and B / S. To create a chart by JFreeChart, it is necessary to complete the following steps. First, store all the data in a container, which is mainly realized by creating a data source Dataset, and all the data queried will be stored in the data source. Second, create a JFreeChart object, which is mainly to import the data from the data source into the object. Finally, set the related properties in JfreeChart object, including chart title, coordinate axis label, chart legend, and curve color. After the above steps are completed, the query data can be displayed (Durresi, Subashi, Durresi, Barolli, \& Uchida, 2019). For example, display a certain environmental parameter value of each hour in a day at a node queried. Figure 7 shows the soil moisture curve of 080F0206 time node queried.

\subsection{System Application Test and Result Analysis}

Test equipment: Seven sensor nodes and one PC accessed to Internet. Through the system setting, one node is used as the gateway node to complete the data aggregation function; two nodes are used as the routing node to complete the routing function; the other four nodes are used as the sensor node to complete the data collection function.

Test method: place all sensor nodes in a certain area of the test farmland so that two sensor nodes can communicate directly with the gateway node, and the other two sensor nodes can communicate with the gateway node only after routing. After the above process is configured, turn on the sensor node to conduct networking communication, and check the data through the serial port debugging assistant on the PC, as shown in Figure 8.

From the interface of serial debugging assistant, it can be seen that there is data written to the serial device. The first four instructions are: instruction 010001261E131E32DD7E; instruction 2, 0100022628132832DD7E; instruction 3, 010003261E131E32DD7E; instruction 4, 0100042632131E32DD7E. According to the communication protocol of the node, the instruction format is composed of five parts, each part represents different meanings, including data header, 
Table 4. History information of sensor node

\begin{tabular}{|c|c|c|c|c|}
\hline Node address & Soil temperature $\left({ }^{\circ} \mathbf{C}\right)$ & Soil moisture (\%RH) & $\mathrm{CO}_{2}$ concentration $(\mathrm{ppm})$ & pH \\
\hline 080F0205 & 12.8 & 74.43 & 385 & 6.5 \\
\hline 080F0205 & 10.3 & 65.53 & 364 & 6.3 \\
\hline 080F0205 & 10.8 & 70.49 & 382 & 6.6 \\
\hline 080F0205 & 10.5 & 78.46 & 380 & 6.0 \\
\hline 080F0205 & 9.4 & 74.56 & 365 & 6.9 \\
\hline 080F0205 & 10.8 & 73.87 & 367 & 6.5 \\
\hline 080F0205 & 11.4 & 70.40 & 335 & 6.8 \\
\hline 080F0205 & 11.6 & 74.47 & 345 & 6.7 \\
\hline 080F0205 & 10.9 & 68.83 & 376 & 6.9 \\
\hline 080F0205 & 11.4 & 65.86 & 364 & 6.4 \\
\hline 080F0205 & 9.4 & 69.41 & 369 & 6.5 \\
\hline 080F0205 & 9.6 & 70.49 & 371 & 6.8 \\
\hline 080F0205 & 9.4 & 71.56 & 387 & 6.4 \\
\hline 080F0205 & 9.2 & 74.56 & 386 & 6.6 \\
\hline 080F0205 & 9.8 & 75.43 & 389 & 6.9 \\
\hline 080F0205 & 10.3 & 74.67 & 384 & 6.7 \\
\hline 080F0205 & 11.2 & 72.45 & 366 & 6.4 \\
\hline 080F0205 & 11.9 & 74.43 & 375 & 6.0 \\
\hline 080F0205 & 12.5 & 73.49 & 367 & 6.3 \\
\hline 080F0205 & 12.6 & 72.41 & 364 & 6.6 \\
\hline
\end{tabular}

Figure 7. Query results of node soil moisture

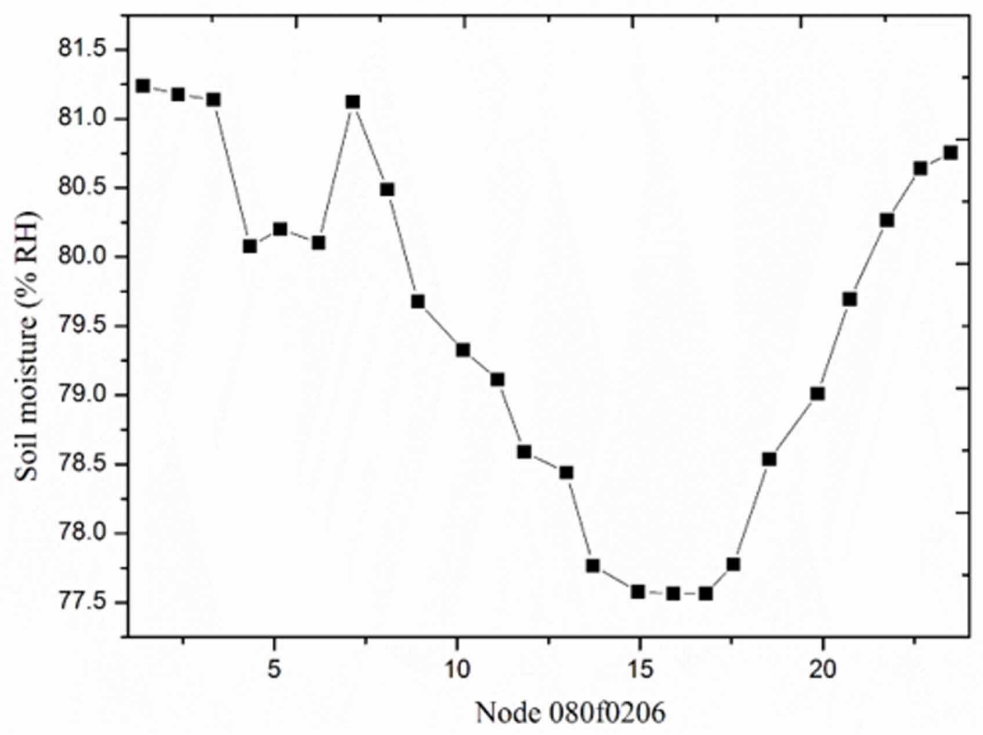


Figure 8. Serial data display

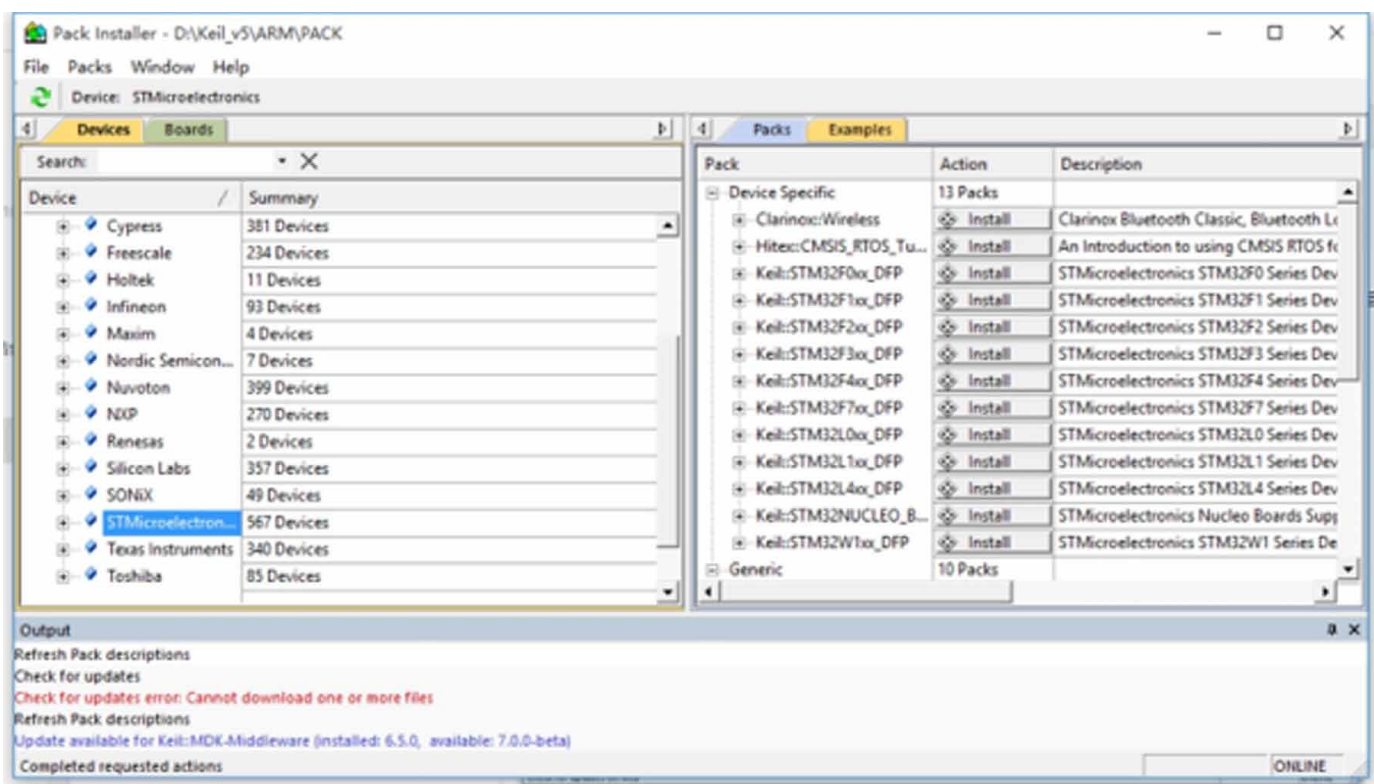

node address, data, check bit, and data tail. For the above instruction 1, it is designed according to the format of data frame sent and received by the serial port, where 01 represents the data header, 0001 represents the node address, 261E131E represents the data, 32DD represents the check bit, and 7 represents the data tail. The above results show that the node networking is successful, the data can be transmitted normally, and the wireless sensor network is running well.

Through the farmland meteorological data and real-time monitoring screen displayed by the monitoring center, agricultural managers can grasp the environmental parameters and growth conditions of crops in real-time, which provides effective basis for the implementation of intelligent control and scientific management of agricultural ecological information, and greatly improves the agricultural production efficiency and management level.

The proposed system for smart agriculture senses temperature, humidity and soil moisture. In this system the sensors are deployed in environment to collect environmental parameters. The proposed system provide more advantages for farmers and can manage time, energy and cost parameters. The measured data is transmitted to server through gateway for the analyses of data in real time. The water resource for the agriculture must be utilized properly as water issues are one of the major problem for irrigation. The proposed system provides the efficient use of water considering a farm of size $200 * 200$ meters. The considered farm is further divided into 4 regions for measuring regional parameters. In each region the sensor nodes are deployed equally and the obtained data at every one hour is transmitted through gateway and analysed. The sensed data is stored in cloud platform, where it can be accessed by end users. The end user can monitors the information of every region at each hour periodically. The obtained results of the system which are presented to the farmers or users are shown in Figure 9.

The user with timely provided information can prepare their fields for harvesting, planting and irrigating purposes. This provided information enables farmers to understand the behaviour of land. The deployment cost for such system can be costly for its initial use but with the increase in productivity this cost will be offset. The system help farmers for growing more products and applying adequate water and utilizing resources properly. With the evolution IoT people are using more intelligent devices for ease life and better outcomes. In this paper an architecture is proposed for real time agricultural 


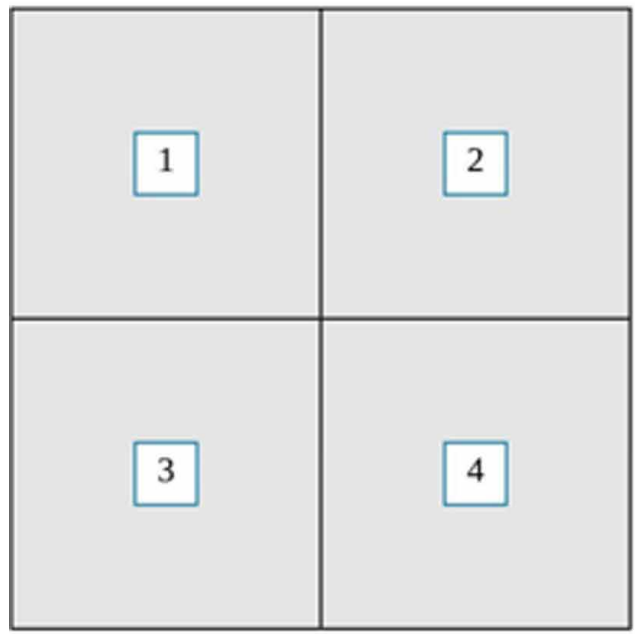

\begin{tabular}{|l|ll|}
\hline & Temp & $28{ }^{\circ} \mathrm{C}$ \\
Region 1 & Humidity & $36 \%$ \\
& Soil Moisture & $38 \%$ \\
& & \\
\hline \multirow{3}{*}{ Region 2 } & Temp & $27^{\circ} \mathrm{C}$ \\
& Humidity & $39 \%$ \\
& Soil Moisture & $45 \%$ \\
\hline \multirow{3}{*}{ Region 3 } & & \\
& Temp & $30^{\circ} \mathrm{C}$ \\
& Humidity & $32 \%$ \\
& Soil Moisture & $40 \%$ \\
\hline & & \\
\hline \multirow{2}{*}{ Region 4 } & Temp & $29{ }^{\circ} \mathrm{C}$ \\
& Humidity & $35 \%$ \\
& Soil Moisture & $40 \%$ \\
& & \\
\hline
\end{tabular}

monitoring through WSN, video surveillance and IoT. The designed system is tested for small and large scale production considering farm land of different sizes with the objective of presenting a user friendly interface. The observed results of the system presents the efficient resource consumption in terms of processing data in real time and making decisions. The user can divide the entire farm land into small regions to get the overall field values. This division of entire field can help farmer to apply irrigation and fertilizers to the desired region only which accordingly increases the overall productivity of crop. Therefore the system is beneficial in providing better saving of time and water.

\section{CONCLUSION AND FUTURE WORK}

In accordance with the practical application requirements of agriculture in China, based on IoT architecture, an agricultural monitoring system that can collect and share information of agricultural environment in real time is designed. The system can collect the environmental parameters of farmland and monitor the growth of crops in real time. The Web server of the monitoring center can store the relevant information collected and provide the basic information base for the development of modern agriculture. Meanwhile, it can publish and share information. After registration, agricultural managers can log in to the system and have real-time understanding of farmland environmental parameters and crop growth conditions. They can also realize data query, download, and node control functions. Based on the above results, it can be concluded that the agricultural monitoring system based on IoT is very important for the development of intelligent agriculture.

In future the prototype of this model will be considered as a deliverable product which will generate real time data and useful for the further development. This model later can be extended with Internet of Drones (IoD) which includes the applications of drones for pesticides spray and crop yielding time. This is also used as crop surveillance equipment. 


\section{REFERENCES}

Anandkumar, V., Kalaiarasan, T., \& Balakrishnan, S. (2018). IoT Based Soil Analysis and Irrigation System. International Journal of Pure and Applied Mathematics, 119(12), 1127-1134.

Anitha, A., \& Acharjya, D. (2018). Crop suitability prediction in Vellore District using rough set on fuzzy approximation space and neural network. Neural Computing \& Applications, 30(12), 3633-3650. doi:10.1007/ s00521-017-2948-1

Awan, S. H., Ahmed, S., \& Hashim, M. Z. (2019). Smart Farm Intelligence Model to Uplift Farmers in Rural Pakistan. International Journal of Wireless Communications and Mobile Computing, 6(4), 49.

Biccario, G., Annese, V. F., Cipriani, S., \& De Venuto, D. (2014). WSN-based near real-time environmental monitoring for shelf life prediction through data processing to improve food safety and certification. Paper presented at the 2014 11th International Conference on Informatics in Control, Automation and Robotics (ICINCO). doi:10.5220/0005102407770782

Bontsema, J., Van Henten, E., Gieling, T. H., \& Swinkels, G. (2011). The effect of sensor errors on production and energy consumption in greenhouse horticulture. Computers and Electronics in Agriculture, 79(1), 63-66. doi:10.1016/j.compag.2011.08.008

Cambra, C., Sendra, S., Lloret, J., \& Garcia, L. (2017). An IoT service-oriented system for agriculture monitoring. Paper presented at the 2017 IEEE International Conference on Communications (ICC). doi:10.1109/ ICC.2017.7996640

Chappell, M., Dove, S. K., van Iersel, M. W., Thomas, P. A., \& Ruter, J. (2013). Implementation of wireless sensor networks for irrigation control in three container nurseries. HortTechnology, 23(6), 747-753. doi:10.21273/ HORTTECH.23.6.747

Chen, M., Mao, S., \& Liu, Y. (2014). Big data: A survey. Mobile Networks and Applications, 19(2), 171-209. doi:10.1007/s11036-013-0489-0

Chen, Y., Zhao, S., \& Zhou, Y. (2018). Research on Intelligent Agricultural Planting System Based on Internet of Things Technology. Journal of Computer and Communications, 6(06), 54-60. doi:10.4236/jcc.2018.66005

Durresi, M., Subashi, A., Durresi, A., Barolli, L., \& Uchida, K. (2019). Secure communication architecture for internet of things using smartphones and multi-access edge computing in environment monitoring. Journal of Ambient Intelligence and Humanized Computing, 10(4), 1631-1640. doi:10.1007/s12652018-0759-6

Guillermo, J. C., García-Cedeño, A., Rivas-Lalaleo, D., Huerta, M., \& Clotet, R. (2018). Iot architecture based on wireless sensor network applied to agricultural monitoring: A case of study of cacao crops in ecuador. Paper presented at the International Conference of ICT for Adapting Agriculture to Climate Change.

Jinbo, C., Yu, Z., \& Lam, A. (2018). Research on monitoring platform of agricultural product circulation efficiency supported by cloud computing. Wireless Personal Communications, 102(4), 3573-3587. doi:10.1007/ s11277-018-5392-3

Khan, S. F., \& Ismail, M. Y. (2018). An Investigation into the Challenges and Opportunities Associated with the Application of Internet of Things (IoT) in the Agricultural Sector-A Review. JCS, 14(2), 132-143. doi:10.3844/ jessp.2018.132.143

Kiani, F., \& Seyyedabbasi, A. (2018). Wireless sensor network and internet of things in precision agriculture. International Journal of Advanced Computer Science and Applications, 9(8), 220-226. doi:10.14569/ IJACSA.2018.090614

Köksal, Ö., \& Tekinerdogan, B. (2019). Architecture design approach for IoT-based farm management information systems. Precision Agriculture, 20(5), 926-958. doi:10.1007/s11119-018-09624-8

Kumar, D., Sharma, A., Kumar, R., \& Sharma, N. (2020). A Holistic Survey on Disaster and Disruption in Optical Communication Network. Recent Advances in Electrical \& Electronic Engineering (Formerly Recent Patents on Electrical \& Electronic Engineering), 13(2), 130-135. 
Lin, S., Li, S., Feng, Q., \& Zou, T. (2018). Research and Implementation of a Modern Agricultural Greenhouse Cultivation System Based on Internet of Things. International Journal of Information Technology and Web Engineering, 13(1), 39-49. doi:10.4018/IJITWE.2018010103

López, J. A., Garcia-Sanchez, A.-J., Soto, F., Iborra, A., Garcia-Sanchez, F., \& Garcia-Haro, J. (2011). Design and validation of a wireless sensor network architecture for precision horticulture applications. Precision Agriculture, 12(2), 280-295. doi:10.1007/s11119-010-9178-1

Marques, G., \& Pitarma, R. (2019). An internet of things-based environmental quality management system to supervise the indoor laboratory conditions. Applied Sciences (Basel, Switzerland), 9(3), 438. doi:10.3390/ app9030438

Myint, C. Z., Gopal, L., \& Aung, Y. L. (2017). WSN-based reconfigurable water quality monitoring system in IoT environment. Paper presented at the 2017 14th International Conference on Electrical Engineering/Electronics, Computer, Telecommunications and Information Technology (ECTI-CON). doi:10.1109/ECTICon.2017.8096345

Pang, Z., Chen, Q., \& Zheng, L. (2011). Scenario-based design of wireless sensor system for food chain visibility and safety. In Advances in computer, communication, control and automation. Springer.

Parameswaran, V., Zhou, H., \& Zhang, Z. (2013). Wireless underground sensor network design for irrigation control: Simulation of RFID deployment. Paper presented at the 2013 Seventh International Conference on Sensing Technology (ICST). doi:10.1109/ICSensT.2013.6727770

Rad, C. R., Hancu, O., Takacs, I. A., \& Olteanu, G. (2015). Smart monitoring of potato crop: A cyber-physical system architecture model in the field of precision agriculture. Agriculture and Agricultural Science Procedia, 6, 73-79. doi:10.1016/j.aaspro.2015.08.041

Rashid, N. K. A. M., \& Bapujee, K. A. (2019). Wireless Wide Area Radiation Monitoring Network Using Internet of Things. Mekatronika, 1(1), 14-20. doi:10.15282/mekatronika.v1i1.978

Ray, P. P. (2017). Internet of things for smart agriculture: Technologies, practices and future direction. Journal of Ambient Intelligence and Smart Environments, 9(4), 395-420. doi:10.3233/AIS-170440

Riquelme, J. L., Soto, F., Suardíaz, J., Sánchez, P., Iborra, A., \& Vera, J. (2009). Wireless sensor networks for precision horticulture in Southern Spain. Computers and Electronics in Agriculture, 68(1), 25-35. doi:10.1016/j. compag.2009.04.006

Ruan, J., Wang, Y., Chan, F. T. S., Hu, X., Zhao, M., Zhu, F., Shi, B., Shi, Y., \& Lin, F. (2019). A life cycle framework of green IoT-based agriculture and its finance, operation, and management issues. IEEE Communications Magazine, 57(3), 90-96. doi:10.1109/MCOM.2019.1800332

Sharma, A., \& Kumar, R. (2016). Performance comparison and detailed study of AODV, DSDV, DSR, TORA and OLSR routing protocols in ad hoc networks. Paper presented at the 2016 Fourth International Conference on Parallel, Distributed and Grid Computing (PDGC). doi:10.1109/PDGC.2016.7913218

Sharma, A., \& Kumar, R. (2017). An optimal routing scheme for critical healthcare HTH services-an IOT perspective. Paper presented at the 2017 Fourth International Conference on Image Information Processing (ICIIP). doi:10.1109/ICIIP.2017.8313784

Sharma, A., Singh, P. K., Sharma, A., \& Kumar, R. (2019). An efficient architecture for the accurate detection and monitoring of an event through the sky. Computer Communications, 148, 115-128. doi:10.1016/j. comcom.2019.09.009

Subashini, M. M., Das, S., Heble, S., Raj, U., \& Karthik, R. (2018). Internet of things based wireless plant sensor for smart farming. Indonesian Journal of Electrical Engineering and Computer Science, 10(2), 456-468. doi:10.11591/ijeecs.v10.i2.pp456-468

Tanumihardja, W. A., \& Gunawan, E. (2015). On the application of IoT: Monitoring of troughs water level using WSN. Paper presented at the 2015 IEEE Conference on Wireless Sensors (ICWiSe).

Vincent, D. R., Deepa, N., Elavarasan, D., Srinivasan, K., Chauhdary, S. H., \& Iwendi, C. (2019). Sensors driven ai-based agriculture recommendation model for assessing land suitability. Sensors (Basel), 19(17), 3667. doi:10.3390/s19173667 PMID:31450772 
Wilkinson, J., \& Rocha, R. (2009). Agro-industry trends, patterns and development impacts. Agroindustries for Development. CABI for FAO and UNIDO.

Yang, R., Liu, Y., Long, H., \& Qiao, L. (2015). Spatio-temporal characteristics of rural settlements and land use in the Bohai Rim of China. Journal of Geographical Sciences, 25(5), 559-572. doi:10.1007/s11442-015-1187-6

Zhang, Q., \& Wang, T. (2019). Design of Internet of Things Method for Environmental Monitoring Based on Big Data. Ekoloji, 28(108), 2513-2517.

Zheng, L., \& Yingbanditpong, O. (2019). Design of Environment Temperature and Humidity Acquisition System of Internet of Things Based on Cloud Platform. Journal of Applied Science and Engineering Innovation, 6(1), 9-13.

Hongyu Hu, a member of the communist party of China (CPC) and a native of dong 'an, hunan province, graduated from Hunan University of Science and Technology in June 1994. He works at Yongzhou Vocational and Technical College, deputy director of the computer department, party branch secretary, dean.

Zheng Chen is an associate professor at College of Computer and Information Science, Hunan Institute of Technology. His current research interests mainly focus on industrialization, urbanization, information technology. 